\title{
Enhanced FCGR2A and FCGR3A signaling by HIV viremic controller IgG
}

\author{
Raymond A. Alvarez, ${ }^{1}$ Ana M. Maestre, ${ }^{2}$ Kenneth Law, ${ }^{1}$ Natasha D. Durham, ${ }^{1}$ Maria Ines Barria, ${ }^{1,3}$ \\ Akiko Ishii-Watabe, ${ }^{4}$ Minoru Tada, ${ }^{4}$ Manav Kapoor, ${ }^{5}$ Mathew T. Hotta,, Gabriela Rodriguez-Caprio, \\ Daniel S. Fierer, ${ }^{1}$ Ana Fernandez-Sesma, ${ }^{2}$ Viviana Simon, ${ }^{1,2,6}$ and Benjamin K. Chen ${ }^{1}$ \\ 'Division of Infectious Diseases, Department of Medicine, Immunology Institute, ${ }^{2}$ Department of Microbiology, Icahn \\ School of Medicine at Mount Sinai, New York, New York, USA. '3epartment of Microbiology, University of Concepcion, \\ Concepcion, Chile. ${ }^{4}$ Division of Biological Chemistry and Biologicals, National Institute of Health Sciences, Tokyo, Japan. \\ ${ }^{5}$ Department of Neuroscience, Icahn School of Medicine at Mount Sinai, New York, New York, USA. ${ }^{6}$ The Global Health \\ and Emerging Pathogens Institute, Icahn School of Medicine at Mount Sinai, New York, New York, USA.
}

HIV-1 viremic controllers (VC) spontaneously control infection without antiretroviral treatment. Several studies indicate that IgC Abs from VCs induce enhanced responses from immune effector cells. Since signaling through $\mathrm{Fc}-\gamma$ receptors (FCCRs) modulate these Ab-driven responses, here we examine if enhanced FCCR activation is a common feature of IgC from VCs. Using an infected cell-based system, we observed that VC IgC stimulated greater FCGR2A and FCGR3A activation as compared with noncontrollers, independent of the magnitude of HIV-specific Ab binding or virus neutralization activities. Multivariate regression analysis showed that enhanced FCGR signaling was a significant predictor of VC status as compared with chronically infected patients (CIP) on highly active antiretroviral therapy (HAART). Unsupervised hierarchical clustering of patient IgC functions primarily grouped VC IgC profiles by enhanced FCGR2A, FCGR3A, or dual signaling activity. Our findings demonstrate that enhanced FCCR signaling is a common and significant predictive feature of VC IgC, with VCs displaying a distinct spectrum of FCCR activation profiles. Thus, profiling FCCR activation may provide a useful method for screening and distinguishing protective anti-HIV IgC responses in HIV-infected patients and in monitoring HIV vaccination regimens.

Conflict of Interest: The authors have declared that no conflict of interest exists.

Authorship note: RAA and AMM are co-first authors. RAA and BKC are co-senior authors.

Submitted: April 27, 2016 Accepted: January 6, 2017 Published: February 23, 2017

Reference information: JCI Insight. 2017;2(4):e88226. https:// doi.org/10.1172/jici.insight.88226.

\section{Introduction}

HIV-1 viremic controller (VC) patients represent a unique population of individuals who naturally suppress HIV replication in the absence of highly active antiretroviral therapy (HAART) $(1,2)$. These VC patients can be divided into 2 subgroups made up of long-term nonprogressors (LTNPs) and elite controllers (ECs). Both LTNPs and ECs maintain normal CD4 levels, but ECs inhibit virus replication below detectable levels (50 copies/ml), while LTNPs maintain potent suppression below 1,000 viral copies/ml of blood. Several studies have reported strong Ab-dependent cellular cytotoxicity (ADCC) and Ab-dependent cellmediated viral inhibition (ADCVI) responses in both subgroups of VCs (3-7). These reports suggest that, in addition to associations with favorable HLA, which can promote strong cytotoxic $\mathrm{T}$ cell responses $(8$, 9), Ab-mediated effector cell responses (Ab-MERs) may also contribute to the HIV "controller" phenotype. In the RV144 HIV vaccine trial, a small reduction in HIV acquisition correlated with the induction of nonneutralizing IgG Ab responses that mediate ADCC after controlling for IgA responses $(10,11)$. Given this common feature, further characterization of IgG responses from VCs may yield valuable insights into the mechanisms that underlie potent antiviral $\mathrm{Fc}_{\mathrm{C}}$ effector responses and identify Ab profiles that may be desirable to emulate in HIV vaccine strategies.

Most Ab-MERs are initiated by engagement of $\mathrm{Fc}-\gamma$ receptor (FCGR) activation on the surface of innate immune effector cells (e.g., NK cells, macrophages, monocytes, and DCs) (12, 13). FCGRs are a diverse family of receptors that orchestrate an array of cellular effector functions, such as ADCC, Abdependent cellular phagocytosis (ADCP), and effector cytokine production. Two activating FCGRs that are particularly important to the induction of ADCC, ADCP, and cytokine production are FCGR2A and FCGR3A. These low-affinity FCGRs are activated by receptor cross-linking induced by binding of IgGantigen immune complexes. Recently, Ackerman et al. demonstrated that HIV controller IgG displayed 
A

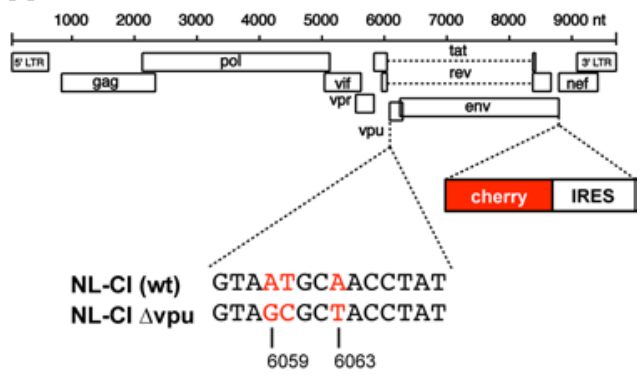

B
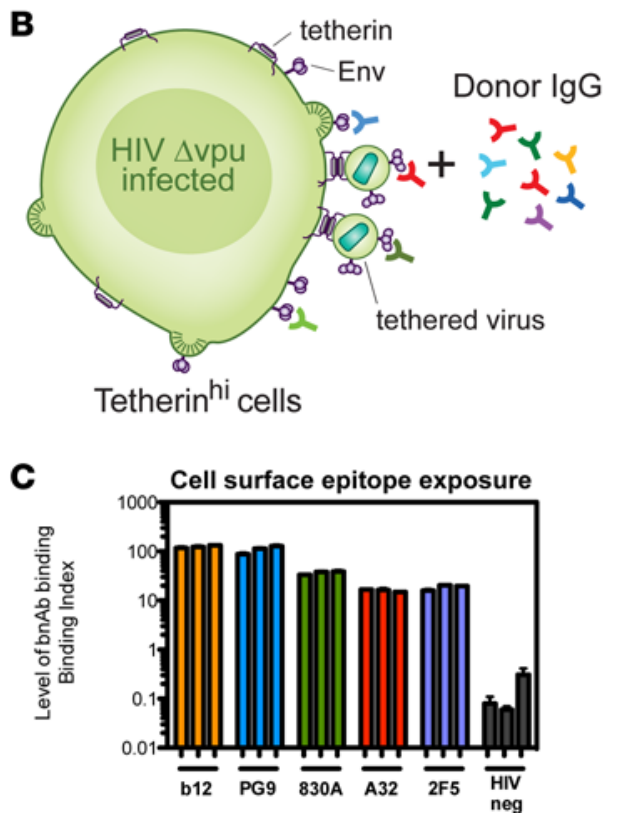

Figure 1. Cell-based HIV-specific Ab binding system. (A) Diagram depicts HIV-1 pNL4.3 construct with mCherry expressed in place of HIV Nef with Nef expression restored by an internal ribosome entry site (IRES), $\Delta$ vpu mutation disrupts the vpu initiation codon. (B) Diagram of HIV Ab binding to tetherin high $\mathrm{CD}^{+}$Jurkat cells infected with an HIV-1 $\Delta$ vpu Cherry-expressing reporter virus. (C) Graph illustrates the reproducibility of epitope detection across 3 separate binding assay replicates using 4 broadly neutralizing monoclonal antibodies (b12; PG9; 830A; 2F5) and non-neutralizing Ab A32, recognizing distinct regions and epitopes within HIV envelope. IgG from an $\mathrm{HIV}^{-}$donor was used as a negative control for binding assay background.

an enhanced breadth of ADCC, ADCP, and NK activation and cytokine production; however, no single Ab-MER was observed to be significantly higher as compared with noncontrollers (14). This result contrasts with other studies that have observed enhanced ADCC/ADCVI activity in controller patients (3-7). Given that FCGR signaling modulates the efficiency of Fc-induced effector responses, assessing the IgG Fc activation profiles associated with controller status may yield insights as to why some VCs display an enhanced breadth of Fc effector responses (14).

IgG Fc effector responses against HIV-1 are dependent on the recognition of the HIV-1 envelope (Env) glycoproteins (gp), since gp120 and gp41 are the only viral antigens exposed on the surface of virions and HIV-infected cells. The form (e.g., virus, cell-surface, or free protein) in which these antigens are presented to antibodies can also greatly influence their ability to activate Fc-mediated responses. Many studies examining $\mathrm{Fc}$ effector responses utilized peptides, monomeric soluble recombinant gp120, or recombinant gp140 trimers (rgp120; rgp140) $(3,6,14-25)$, but these antigens may not reflect the epitopes that are exposed on the conformationally flexible and highly glycosylated native structure of the HIV-1 Env trimer expressed on the surface of HIV-infected T cells (26-29).

Previous studies demonstrate that the antiviral factor tetherin enhances the presentation of viral antigens to the innate immune system through Ab-dependent mechanisms (30-33). Building on these observations, we have developed a tetherinexpressing, cell-based system to measure HIV-specific Ab binding, FCGR signaling, and Ab-MERs using a single platform expressing functional infectious antigens. This system characterizes Abs that recognize epitopes that are exposed on cell-associated and virus incorporated (CANVI) forms of HIV Env. Herein, we utilize this system in conjuction with neutralization assays to examine the levels of HIV-specific binding, FCGR activation, and neutralization mediated by IgG from VCs versus $\mathrm{HIV}^{+}$chronically infected patients (CIPs) on HAART.

\section{Results}

A cell-based Ab binding assay to characterize HIV-specific Abs. Expression of the anti-viral factor tetherin on the surface of HIV-infected $\mathrm{T}$ lymphocytes allows for the enhanced detection of FCGR signaling and FCmediated effector functions (30). To exploit the potential of tetherin to increase the detection of cell-surface viral antigens, we developed an HIV-infected, cell-based system that allows for the characterization of Abs that bind CANVI forms of HIV Env. In this assay system, a subclone of the Jurkat E6 CD4 T cell line, which constitutively expresses high levels of tetherin, is infected with an HIV-1 $\Delta$ vpu mCherry fluorescent reporter virus (Figure 1A). Upon infection, these cells retain virus particles on their surface, along with unincorporated forms of Env expressed on the surface of infected cells (Figure 1B) (30). To quantify $\mathrm{Ab}$ binding, a binding index (BI) was established that provides a combined measure of the frequency of opsonized HIV-infected cells (\%) and the density of HIV-specific Ab binding (median fluorescent intensity [MFI]), since FCGR activation is dependent on both (Supplemental Figure 1; supplemental material available online with this article; https://doi.org/10.1172/jci.insight.88226DS1) (34). For each sample, the $\mathrm{HIV}^{-}$population was used as an internal control to normalize for the contribution of nonspecific $\mathrm{Ab}$ binding in both the frequency of opsonized HIV infected cells (\%) and density of Ab binding (MFI) (Supplemental Figure 1A).

To address the possibility that the overall level and heterogeneity of HIV Env expressed on the surface of infected cells may vary in our system, we first examined the level and reproducibility of epitope exposure among experimental replicates (Figure 1C). For this purpose, tetherin ${ }^{\text {high }} \mathrm{HIV}-1 \Delta \mathrm{vpu}$-infected 
A

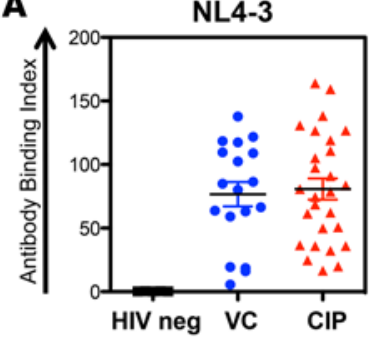

YU2

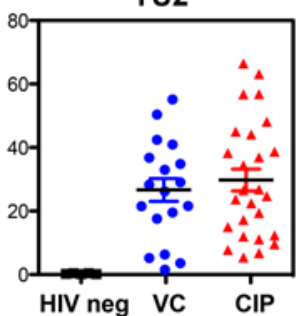

YU2

B
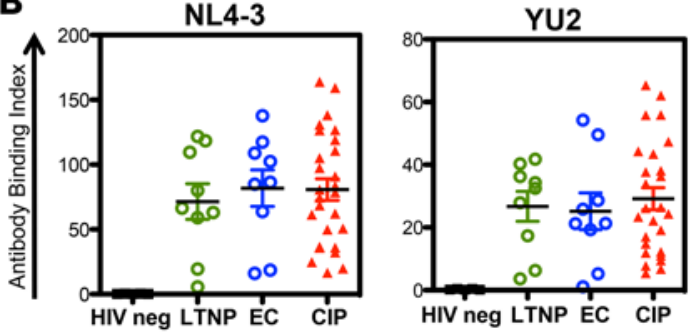

RHPA.c

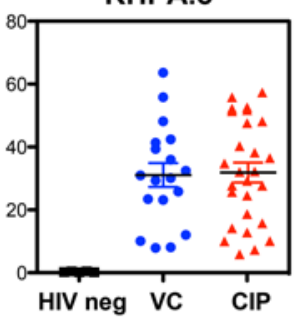

RHPA.c

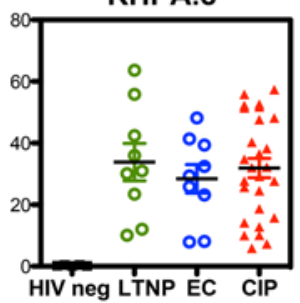

JRFL

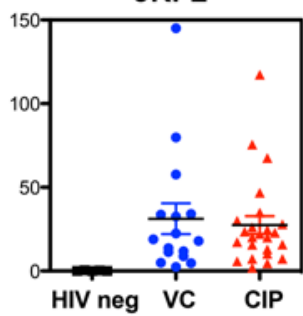

JRFL

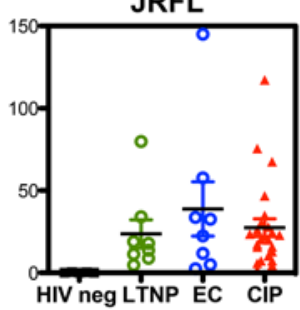

\section{C}

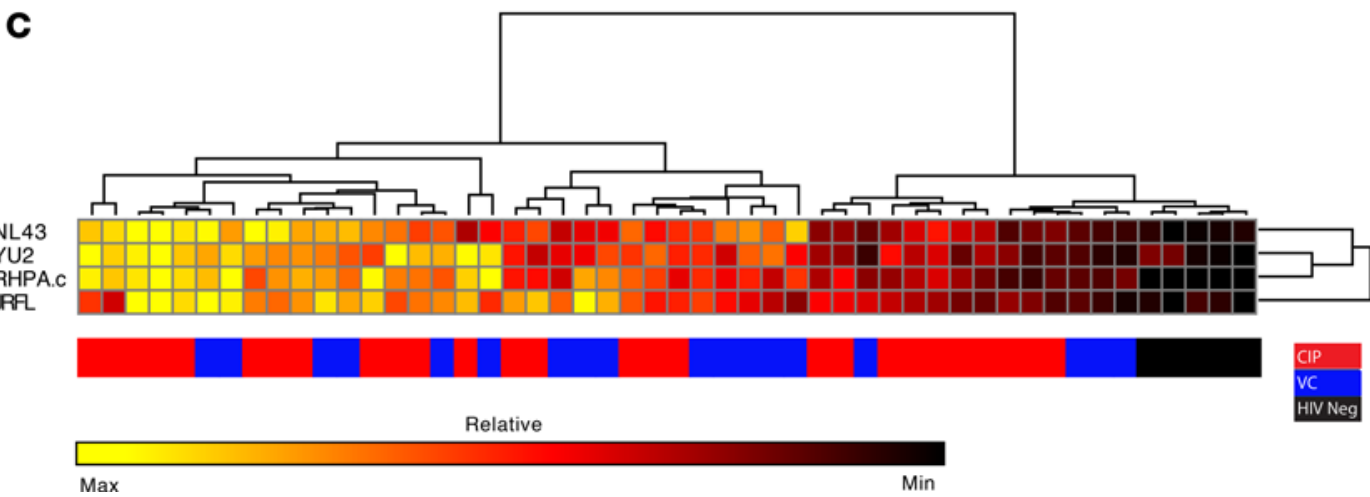

Figure 2. HIV-specific patient IgG binding to cell-associated and virus-incorporated (CANVI) epitopes. (A) Panel of graphs show the binding index values for viral controller (VC) and chronically infected patient (CIP) IgC using 2 HIV molecular clone envelopes (Envs) (pNL4-3 [X4] or YU2 [R5]), transmitted founder (T/F) Env (RHPA.c), or primary R5 Env (JRFL). (B) The binding index values for CIPs vs. VC split into ECs vs. LTNPs. (C) Hierarchical clustering analysis of the relative percentile rank of Ab binding to each HIV-1 Env. Each column in the heat map indicates an individual patient's IgG. Colored bars along the bottom correspond to the IgG patient group. The mean of 3 experiments for $n=50$ IgG is shown.

cells that were $20 \%-30 \%$ infected, as indicated by mCherry expression, were used to measure the level of anti-HIV Ab binding with Abs that recognize different defined epitopes on HIV Env. We observed that the levels of $\mathrm{Ab}$ binding varied depending on the $\mathrm{Ab}$ used, but the levels of Env detection for each $\mathrm{Ab}$ remained consistent across replicates (Figure 1C). This demonstrates relatively consistent exposure of epitopes and Env forms in our system. Importantly, we also tested the CANVI system with 2 Abs that recognize conformational epitopes, $830 \mathrm{~A}$ and PG9 $(35,36)$. Specifically, PG9 has previously been shown to bind a trimer-dependent epitope in the V2 and V3 loops of HIV-1 Env (36). Since PG9 displays $90 \%$ of the BI value of $\mathrm{B} 12$, which had the highest $\mathrm{BI}$ of the broadly neutralizing monoclonal Abs (bnAbs) tested, this provides some evidence that a majority of Env antigen in our system is in a higher-order quaternary structure representative of native Env trimers. Our Ab binding assay contrasts with traditional ELISA methods that use rgp120, do not present native higher order conformational epitopes, and may display epitopes that are masked in native Env trimers.

Quantifying the level of patient IgG HIV-specific Ab binding. We next examined the level of patient Ab binding to HIV-1 Envs using IgG purified from the plasma of $18 \mathrm{HIV}^{+} \mathrm{VC}$ patients, $27 \mathrm{HIV}^{+} \mathrm{CIPs}$ on HAART, and $5 \mathrm{HIV}^{-}$donors (Table 1). The HIV $\mathrm{HC}^{+}$consisted of 2 subgroups, $9 \mathrm{ECs}$ with viral loads $<50$ copies/ $\mathrm{ml}$ and 9 LTNPs with $\mathrm{VL}<1,000$ copies/ml, both of which have controlled infection without antiretroviral therapy (ART). Ab binding was measured using our cell-based CANVI Env system using a panel of X4- or 
Table 1. Clinical characteristics of the study cohort

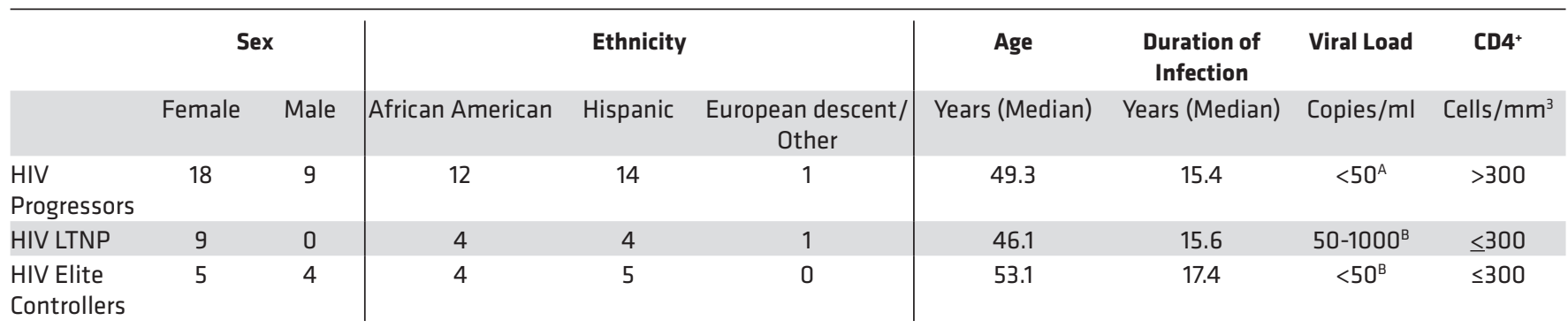

${ }^{\mathrm{A}}$ Received HAART treatment. ${ }^{\mathrm{B}}$ Absence of HAART treatment.

R5-tropic HIV Envs that included 2 molecular clones (NL4-3, YU2), a transmitted founder Env (RHPA), and an Env clinical isolate (JRFL). While we observed a broad range of HIV-specific patient Ab binding, we observed no significant differences in the levels of binding between IgG from VC versus CIP (Figure 2A). Furthermore, we observed no significant differences in comparing the VCs split into EC and LTNP subgroups versus CIP (Figure 2B). NL4-3 Env was the most highly recognized Env across all patient IgG (i.e., the highest absolute BI values compared with other HIV-1 Envs). To examine the degree of similarity and relative magnitudes of $\mathrm{Ab}$ binding for each patient IgG to different HIV Envs, we performed an unbiased hierarchical clustering analysis using Gene E software. This analysis allowed us to examine the degree of similarity and relative magnitudes of $\mathrm{Ab}$ binding for each patient IgG to different HIV Envs, relative to all IgG in the cohort. The heat map indicating the breadth of IgG binding revealed that the IgG with strong binding to NL4-3 also bound well to YU2, JRFL, and RHPA Env (Figure 2C). Additionally, breadth of binding was not preferentially distributed among VCs or CIPs upon cluster analysis (Figure 2C). These data indicate that the level and breadth of HIV Env recognition do not associate with controller status.

A

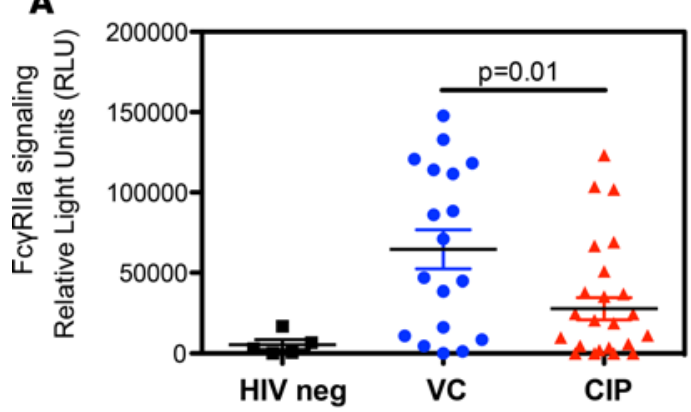

C

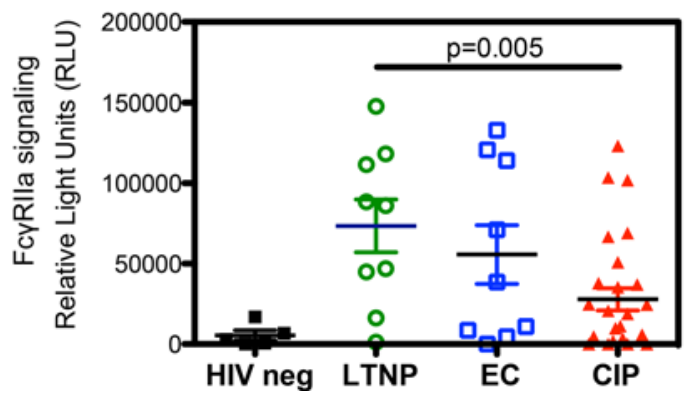

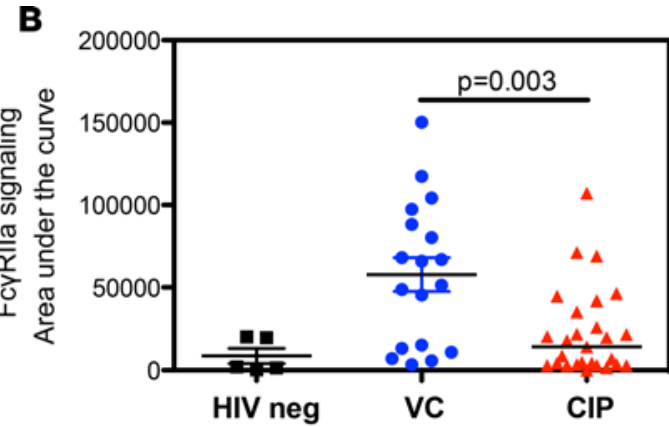

D

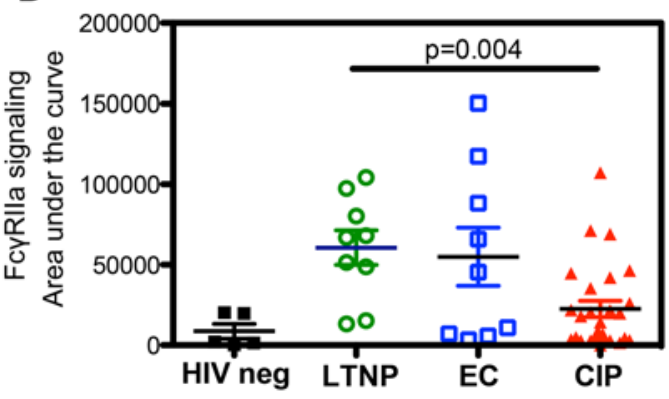

Figure 3. FCGR2A signaling in response to HIV+ $^{+}$patient IgC Abs. Graphs depict the levels of FCGR2A signaling mediated by $\mathrm{HIV}^{+}$patient lgG opsonized HIV-1 $\Delta$ vpu-infected target cells. For FCGR signaling experiments, Abs were diluted 10-fold, starting at a top concentration of $250 \mu \mathrm{g} / \mathrm{ml}$ in a 5-point titration curve. (A and C) Maximum signals achieved at the top concentration of $A$ bs tested $(250 \mu \mathrm{g} / \mathrm{ml})$. (B and $\mathbf{D})$ The area under the signaling curve. (A and B) Comparison of VC vs. CIP. (C and D) Comparison of LTNPs, ECs, and CIPs. The mean of 3 experiments for $n=50$ IgC is shown. 
A

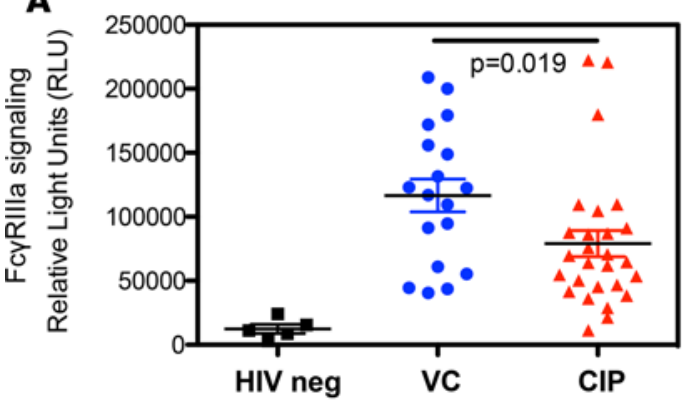

C

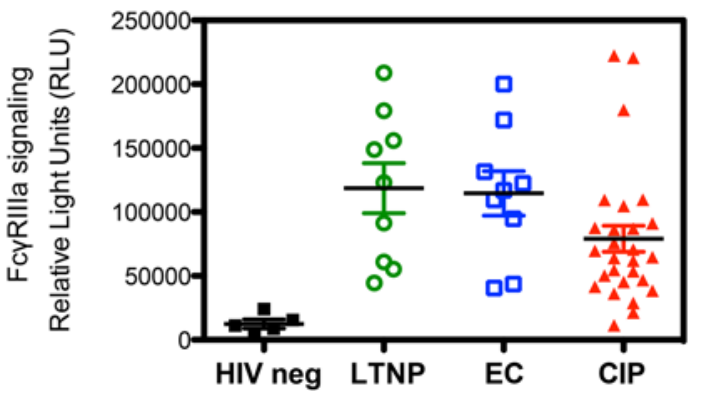

B

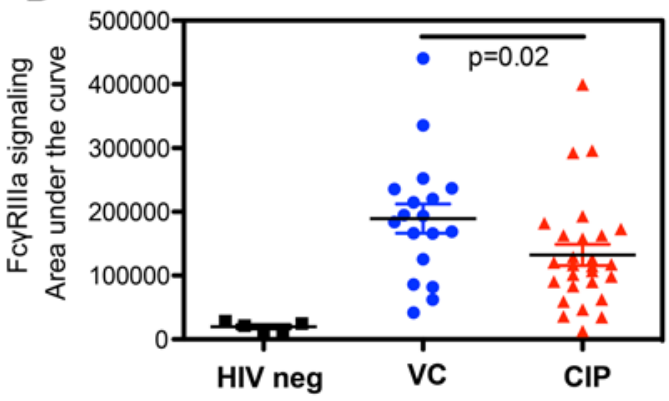

D

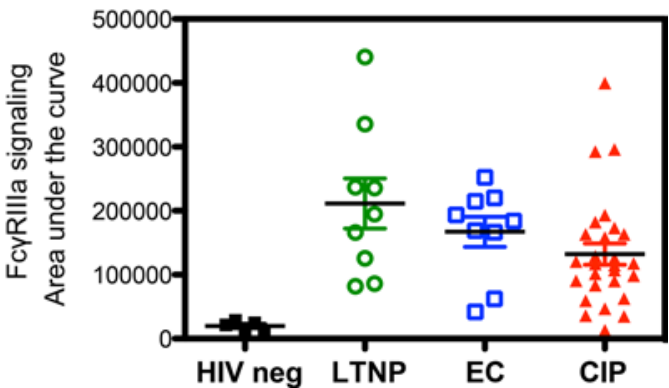

Figure 4. FCGR3A signaling and FCGR hierarchical clustering analysis in response to HIV+ patient IgC Abs. Graphs depict the levels of FCGR3A signaling mediated by HIV-1 $\Delta$ vpu-infected target cells opsonized with HIV+ patient IgG. For FCCR signaling experiments, Abs were diluted 10 -fold, starting at a top concentration of $250 \mu \mathrm{g} / \mathrm{ml}$ in a 5-point titration curve. (A and C) The maximum signals achieved at the top concentration of Abs tested $(250 \mu \mathrm{g} / \mathrm{ml})$. (B and $\mathbf{D})$ The area under the signaling curve. (A and B) Comparison of VC vs. CIP. (C and D) Comparison of LTNPs, ECs, and CIPs. The mean of 3 experiments for $n=50$ IgC is shown.

VCs possess enhanced FCGR activation. A number of studies describe the capacity of IgG from VCs to mediate enhanced ADCC and ADCVI (3-7), or enhanced polyfunctional Fc-mediated responses (14); however, the FCGR signaling profiles associated with these activities have not yet been characterized. To examine FCGR signaling, we utilized our CANVI Env system, coculturing these cells with either an FCGR2A or FCGR3A reporter cell line that induces nuclear factor of activated T cell-driven (NFAT-driven) luciferase expression in response to FCGR activation $(30,37)$. For this assay, HIV $\Delta v$ vu-infected tetherin ${ }^{\text {high }}$ cells were treated with a serial dilution of patient IgG Abs and cocultured with either FCGR2A or FCGR3A reporter cells.

When examined for FCGR activation, VC Abs mediated enhanced signaling as compared with $\mathrm{CIP}$ Abs at the highest Ab concentration tested for both FCGR2A (Figure 3A) and FCGR3A (Figure 4A). Enhanced FCGR signaling by VC Abs was also apparent when comparing the total areas under the FCGR signaling curves (Figure 3B and Figure 4B). Importantly, in splitting the VCs into LTNPs and ECs, the enhanced FCGR activation was equally distributed among both subgroups, indicating that enhanced FCGR activation is a common correlate in both ECs and LTNPs (Figure 3, C and D; Figure 4, C and D).

Anti-HIV IgG from HIV VC and CIP do not display differences in HIV-1 neutralization capacity. To investigate the relationship between the capacity of patient IgG to mediate FCGR signaling and neutralization, we assessed the capacity of each IgG sample to neutralize the same HIV-1 Env used to assess FCGR signaling. Each patient IgG was tested for its ability to neutralize infection of the TZM-bl indicator cell line (38). HIV-1 was preincubated with serial dilutions of patient IgG before exposing the cells to the virus. IgG from $\mathrm{HIV}^{-}$donors were used as negative controls (Figure 5A). The bnAb $2 \mathrm{G} 12$ (data not shown) and pooled polyclonal anti-HIV IgG were used as positive controls (Figure $5 \mathrm{~B}$ ). We observed no significant difference in capacity of VC versus CIP Abs to neutralize HIV-1, either at the highest Ab concentration tested (Figure $5 \mathrm{C}$ ) or in comparing the total areas under the neutralization curves (Figure 5E). Further, we observed no significant difference in virus neutralization activity between CIP and VC when the data was split into EC and LTNP subgroups (Figure 5, D and F). Only 8 of 18 VCs and 10 of 27 CIPs achieved a 50\% tissue culture infective dose $\left(\mathrm{TCID}_{50}\right)$ within the range of $\mathrm{Ab}$ concentrations tested (data not shown). 
A

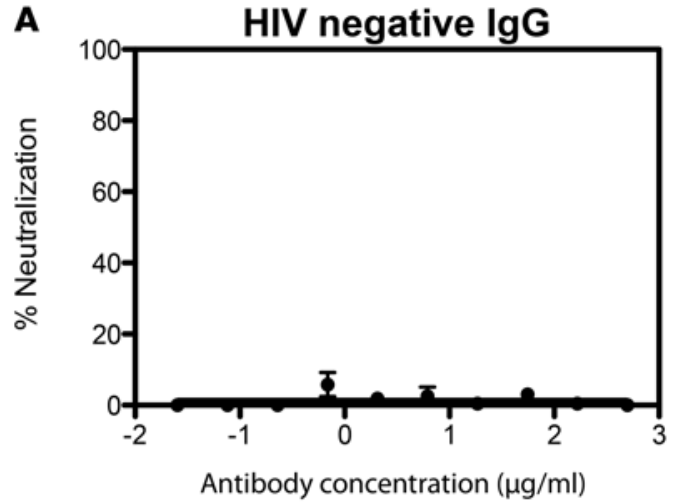

C

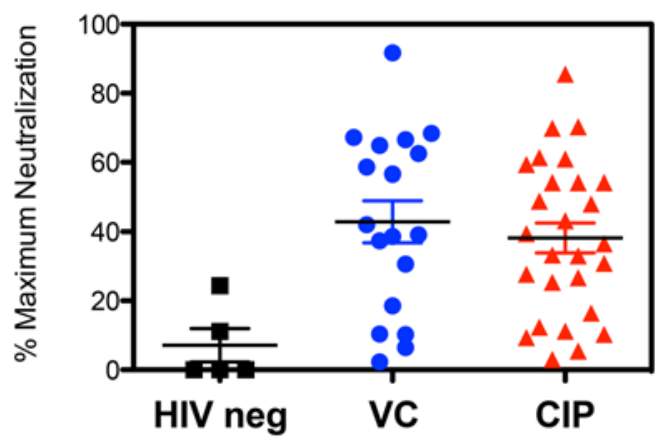

$\mathbf{E}$

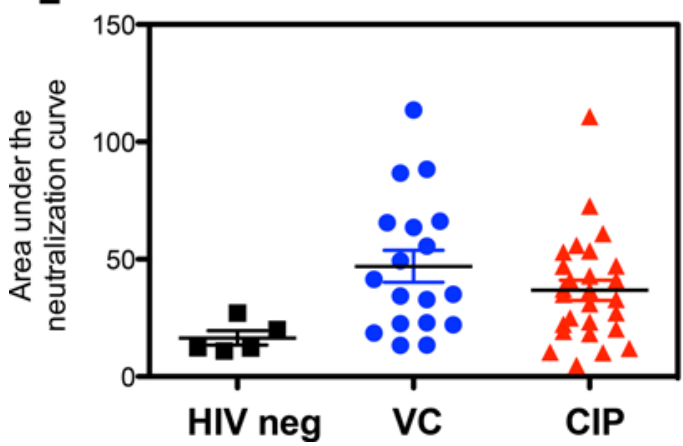

B

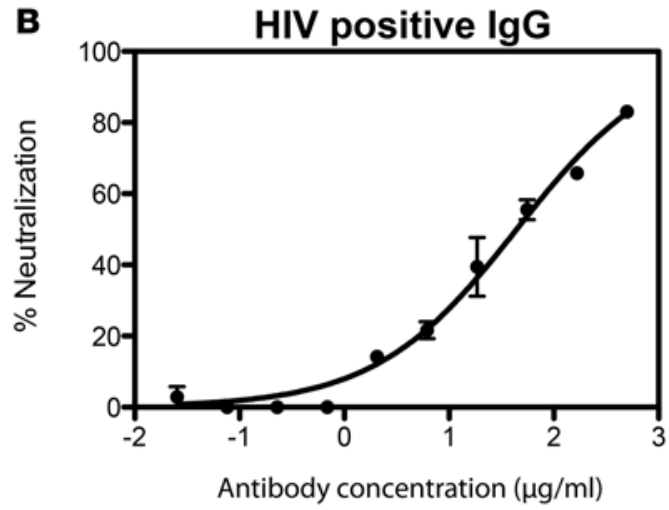

D

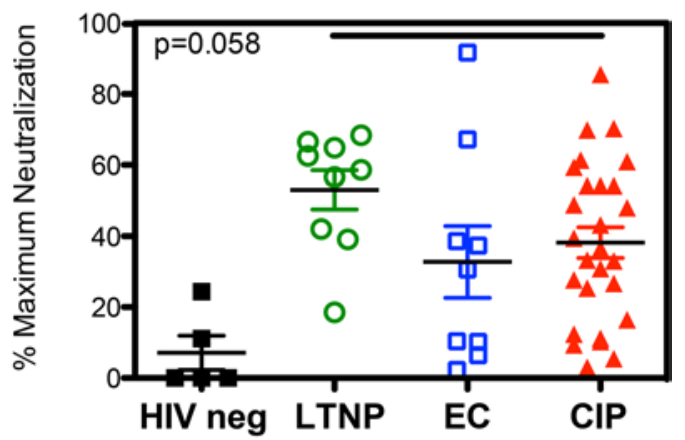

$\mathbf{F}$

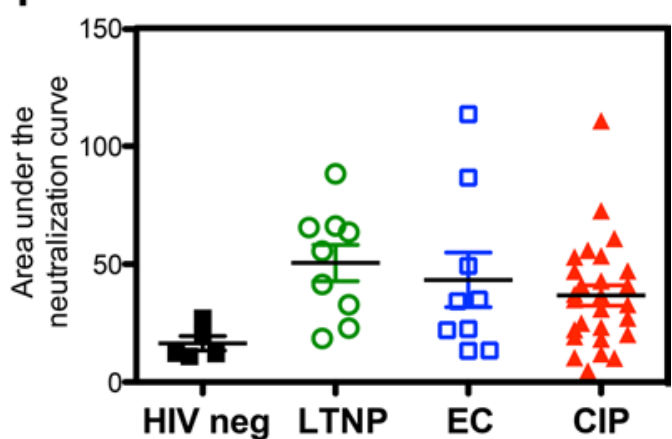

Figure 5. IgG from viremic controllers (VCs) and chronically infected patients (CIPs) do not differ in their HIV-1 neutralizing activity. (A and B) Controls for HIV-1 neutralization assay carried out using TZM-bl cells infected with X4-tropic HIV-1 (pNL4-3). Neutralization curve for (A) polyclonal HIV- IgG sample or (B) polyclonal HIV+ IgG sample are shown. For HIV-1 neutralization assays, polyclonal IgG were diluted 3-fold starting from a top final concentration of $500 \mu \mathrm{g} / \mathrm{ml}$ in a 10 -point titration curve. (C) Maximum neutralization activity of patient-derived IgG comparing noninfected (HIV neg) with VC and CIP. (D) Maximum neutralization activity with VCs split into LTNP and EC subgroups. (E) Neutralization activity as measured by the area under the neutralization curve (AUC) for IgG purified from CIP, VC, and noninfected donors (HIV neg). (F) Neutralization activity (AUC) with VCs split into LTNP and EC subgroups. The mean of 3 experiments for $n=50$ IgG is shown.

Multiparameter analysis indicates distinct VC versus CIP phenotypes/profiles. We next compared the magnitude and relationship of multiple $\mathrm{Ab}$ features across the cohort. A comparison of the levels of HIV-specific cellbased Ab binding to the levels of FCGR2A or FCGR3A activation revealed no significant correlations (Figure 6, A and B). This suggests that the efficiency of FCGR signaling is not solely dependent on the level of $\mathrm{Ab}$ binding to HIV Env. This further implies that VC IgG are qualitatively different from CIP IgG in their ability to mediate FCGR signaling, but they are not quantitatively different in their ability to bind to infected cells.

Next, we compared the levels of Ab-mediated FCGR signaling with neutralization efficiency. We observed no significant correlation between FCGR2A signaling and HIV-1 neutralization (Figure 6C). Interestingly, FCGR3A signaling correlated with neutralization only in CIPs $(P=0.003)$ but not in VC 
A

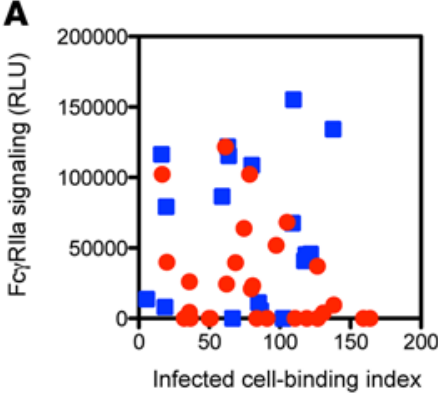

B

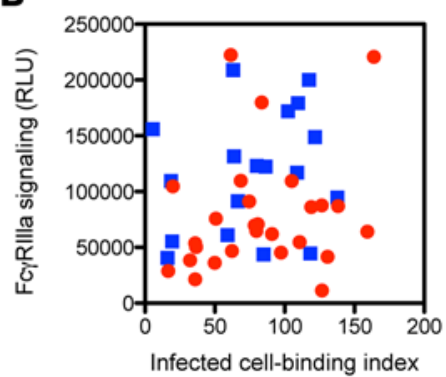

C

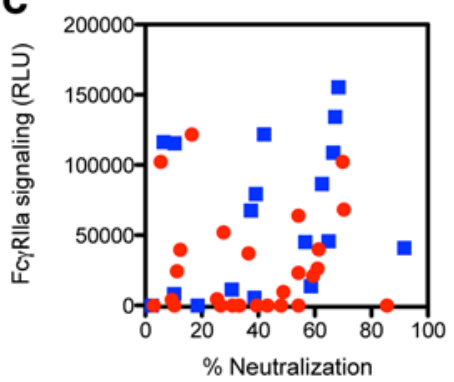

D

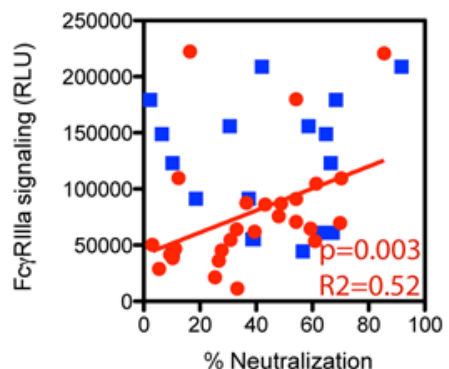

E

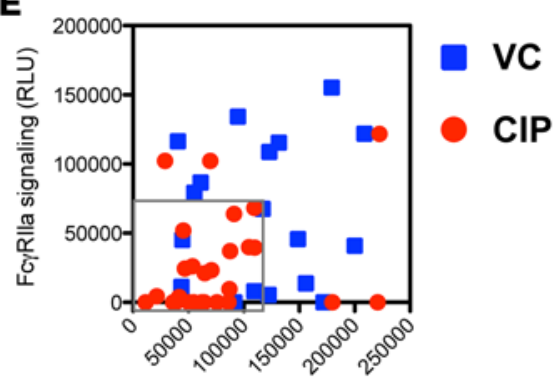

FCүRIIla signaling (RLU)

\section{$\mathbf{F}$}

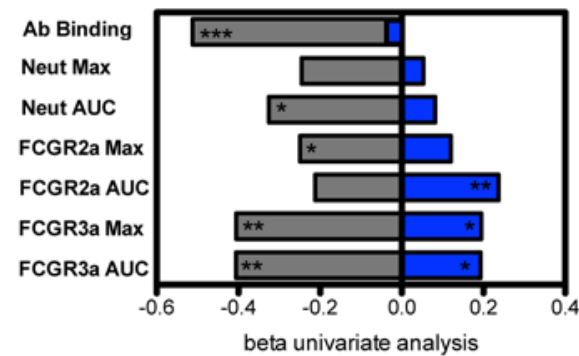

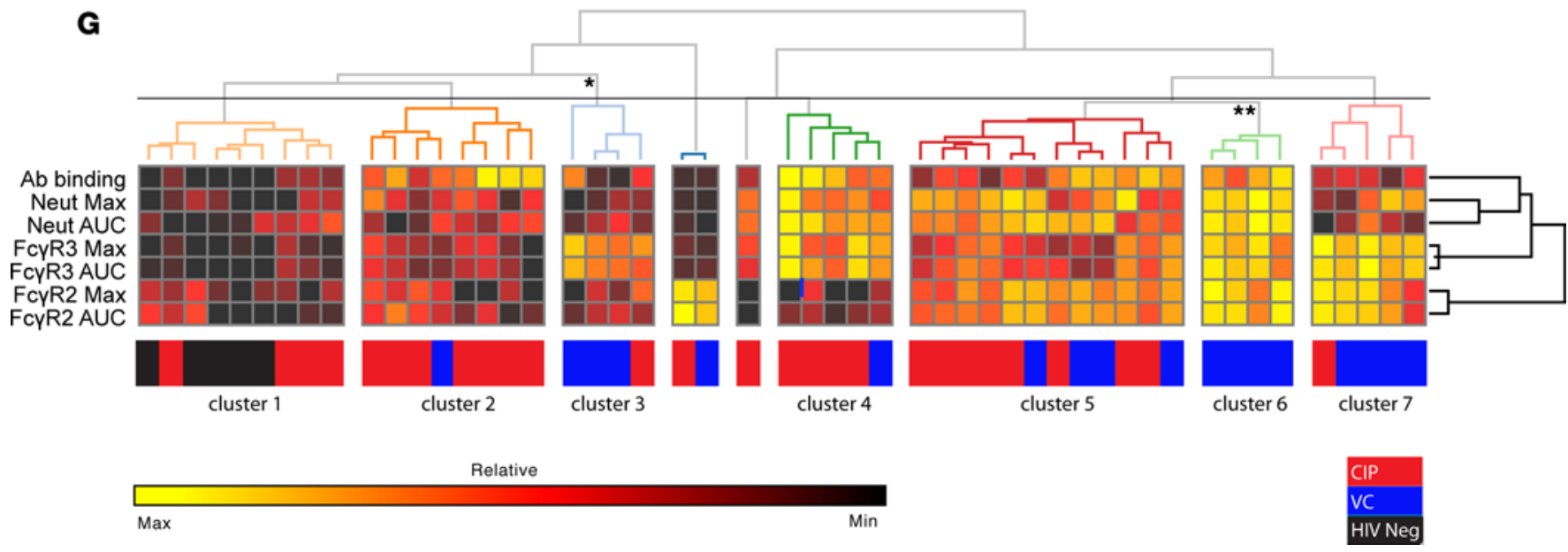

Figure 6. Multiple parameter analysis. (A-E) Graphs show correlations across each pair of independently assessed Ab functions in VC and CIP using Spearman's rank correlations. (F) The cumulative $\beta$ deviation of VCs (blue) or HIV- (gray) from CIPs, which are represented by the vertical line at 0.0 . (G) Hierarchical clustering of patient IgG profiles using a dendrogram heat map, incorporating Ab binding, neutralization, and FCGR signaling. The heat map displays the percentile rank of Ab parameters for each patient. Colored bars along the bottom define IgG parameter that correspond to patient group. ${ }^{*} P>0.05,{ }^{* *} P>0.01,{ }^{* *} P>0.005$. Significance of patients IgG cluster profiles of greater then 3 patients are shown. For univariate and multivariate analysis, $P$ values were calculated using MANOVA in R using the Hotelling-Lawley analysis to find the significance of the model. Correlations between the interrelatedness of $2 \mathrm{Ab}$ functions were calculated using Spearman rank tests. Clustering of patients and IgG properties were based on pair-wise Euclidean distance measurements. Significance was calculated using the multiscale bootstrap resampling.

$(P=0.45)$ (Figure 6D). This suggests that, in CIP, the efficiency of virus neutralization is associated with the efficiency of FCGR3A signaling.

Additionally, we examined the relationship between the levels of FCGR2A versus FCGR3A activation. In doing so, we observed that VCs displayed a spectrum of FCGR activation profiles (Figure 6E). The scatter plot shows that $\sim 80 \%$ of VCs achieved higher signaling for 1 or both FCGRs (out of box, Figure $6 \mathrm{E}$ ). The spectrum of FCGR activation profiles among VC IgG suggests that the magnitude and types of FCGR effector responses elicited by these IgG will differ, since FCGR expression profiles differ among effector cell populations (e.g., monocyte, macrophages, DCs) $(12,13)$. In contrast to VCs, $\sim 80 \%$ of CIPs had lower FCGR2A and FCGR3A signaling as defined by about $<100,000$ RLU for FCGR3A signaling and $<70,000$ RLU for FCGR2A signaling (within box, Figure 6E). 
To assess how strongly $\mathrm{Ab}$ functions can predict controller or noncontroller status, we conducted a univariate regression analysis comparing variations in $\mathrm{IgG}$ function between the different patient groups. Figure $6 \mathrm{~F}$ displays a univariate regression analysis of IgG function values from $\mathrm{VC}$ or $\mathrm{HIV}^{-}$populations, relative to CIP. High FCGR2a AUC, FCGR3a AUC, and FCGR3a max were each significantly predictive of VC status versus CIP status (Figure 6F). Multivariate regression analysis using all IgG functions demonstrated that, cumulatively, they were able to significantly predict patient group $(P=0.036)$. However, conducting multivariate analysis with just the variables that most significantly distinguished all patient groups (FCGR2a AUC, FCGR3a AUC, and FGR3a), we observed that this was a more significant predictor of patient group, including controller status $(P=0.00018)$.

In addition, we compared all of the $\mathrm{Ab}$ parameters tested using unbiased hierarchical clustering of all metrics. Figure $6 \mathrm{G}$ displays a dendrogram heat map of all IgG parameters tested for each patient, where the dendrogram shows the degree of similarity or clustering between patients and IgG functions. Using this approach, we observed that VCs were primarily enriched in distinct IgG profile clusters based predominently on high, HIV-specific FCGR signaling. Cluster 7 contained $4 \mathrm{VCs}$ out of $5 \mathrm{HIV}^{+}$patients and was defined by high dual FCGR activation in the absence of high Ab binding or neutralization. Cluster 6 consisted solely of 4 VCs that displayed a profile of uniformly high Ab binding, neutralization, and FCGR activation. Finally, cluster 3, which consisted of $3 \mathrm{VCs}$ out of $4 \mathrm{HIV}^{+}$patients, was characterized by high FCGR3a activation in the absence of high Ab binding, neutralization, or FCGR2a activation. Overall, these VC clusters were primarily defined by high levels of dual or single FCGR activation ( 11 of $18 \mathrm{VCs}$ ). In examining the significance of clusters using R package "pvclust" (39), we observed that VC enriched clusters 3 and 6 were significantly distinct from other IgG profiles.

Examining the effect of ART and CD4 count on IgG-induced functions. A caveat in studying CIP as a comparator group is the potential for ART to modulate HIV-specific Ab titers, CTL response, or neutralizing activity (40-43). A recent longitudinal study by Madhavi et al. found a $35 \%$ reduction in the ADCC activity in ART-treated patients (44). We therefore examined how the duration of ART within CIP may influence the Ab parameters tested in this study. The CIP in this study were on ART for 6 months up to 10 years. When we compared the length of time on ART versus FCGR signaling, virus neutralization or $\mathrm{Ab}$ binding, we observed no significant correlations with time on ART (Supplemental Figure 2). Therefore, the duration of time that CIPs were on ART did not affect the mean distribution of any $\mathrm{Ab}$ parameters and is not likely to be a major confounder in this study.

In addition, non-HIV and HIV-specific IgG responses in $\mathrm{HIV}^{+}$patients on HAART have been correlated with CD4 count $(41,42,45,46)$. Therefore, we also examined if CIP CD4 cell counts at time of sample collection were correlated with the efficiency of HIV-specific IgG functions. CD4 counts for CIP ranged from 277-1,297, and in comparing CD4 cell counts versus time on HAART, we noted no significant correlations (Supplemental Figure 3A). In comparing the CD4 counts versus HIV-specific Ab functions, we also observed no significant correlations (Supplemental Figure 3, B-D). Therefore, CD4 counts at the time of sample collection did not correlate with the efficiency of IgG-mediated responses in this cohort.

\section{Discussion}

Many studies indicate that $\mathrm{Ab}$-induced effector responses mediated through FCGR signaling contribute to the control and prevention of HIV-1 infection (3-7, 10, 11, 14, 47-50). Here, we set out to define the profile of FCGR activation associated with potent control of HIV-1 and examine how FCGR activation was more broadly associated with other IgG functions (e.g., Ab binding, neutralization). In addition, we aimed to develop the quantitative assays that can assess the functional properties of antibodies in HIV controller patients, as well as those elicited by therapeutic or prophylactic HIV vaccines.

A key parameter in assessing functional HIV-specific Ab responses is the optimal selection of capture antigens that reflect the epitopes that are accessible on the conformationally flexible and highly glycosylated Env trimer. The characterization of Abs that recognize exposed epitopes is particularly important, since Abs that recognize Env expressed on the surface of infected cells and incorporated into virus particles may represent the Abs that trigger Fc effector responses against infected cells in vivo. Many studies examining Fc-mediated effector responses (i.e., ADCC) use recombinant protein or peptide-pulsed cells as targets $(3,6,10,14-25,47)$; however, for the reasons stated above, these may not reflect the epitopes available on the surface of infected cells or in virions. Indeed, a recent study observed that ADCC induced by HIV ${ }^{+}$ patient-derived Abs was significantly higher when using rgp120 as the capture antigen, as compared with a scaffolded trimeric form of Env using the same sera (51). 
In studies that have utilized infected cells to characterize anti-HIV antibodies, the relatively low level of Env expression maintained on the cell surface of infected cells, due to active endocytosis and shedding $(52,53)$, may limit the efficiency of IgG opsonization of infected cells. Thus, these assays may not achieve sufficient sensitivity to discriminate different IgG-induced Fc effector responses. For these reasons, we developed a cell-based $\mathrm{Ab}$ binding assay system that allows for the enhanced detection of Fc-mediated effector responses elicited by Abs that bind CANVI forms of HIV Env. Importantly, this system enables quantification of multiple Fc effector responses using a single platform and allows for direct functional testing of their Fc-signaling capacities. The CANVI system provides consistency when comparing $\mathrm{Ab}$ binding, $\mathrm{Fc}$ signaling, and $\mathrm{Fc}$ effector functions, since the form of HIV capture antigen remains consistent across different assays.

In this study, we utilized our CANVI Env system to examine the level and breadth of patient IgG $\mathrm{Ab}$ binding to various HIV-1 Envs. We observed no significant correlation with the absolute level or breadth of binding and HIV controller status (Figure 2). The CXCR4 HIV-1 Env, NL4-3, yielded the highest absolute level and relative differences in anti-HIV IgG binding between samples, as compared with R5-tropic lab isolate YU2 Env, primary T/F RHPA Env, or clinical isolate JRFL Env. Therefore, we utilized NL4-3 Env to examine the efficiency of FCGR signaling mediated by VC or CIP IgG.

In examining the level of FCGR signaling mediated by VC or CIP IgG, we observed that VC IgG possessed an enhanced capacity to mediate FCGR2A and FCGR3A activation (Figure 3, A-D). In subdividing the VCs into ECs and LTNPs, we still observed some of the significant differences in FCGR signaling, despite smaller patient numbers. In spite of examining a modestly sized controller cohort, the discrimination of significant signaling differences in the different patient groups highlights that enhanced FCGR signaling may be a common IgG function that underlies the potent control of HIV infection in VCs.

When directly comparing the level of FCGR activation versus the level of Ab binding for each patient (Figure 5, A and B), we observed no significant correlations. These data suggest that the level of Ab binding is not associated with the level of FCGR activation induced by either VC- or CIP-derived Abs. This is in contrast with previous studies that have detected higher titers of HIV-specific ADCC Abs in VCs (3) and observed that higher levels of Ab opsonization of infected cells led to higher levels of ADCC (29). In the first of these 2 studies, the use of gp120 ELISA for detection of HIV-specific titer may overestimate $\mathrm{HIV}$-specific $\mathrm{Ab}$ titers (3). In the second study, the level of Ab opsonization was examined on infected cells, but patient samples were pooled, potentially obscuring the relationship between Ab binding and ADCC (29). More recent studies have not found higher HIV-specific titer differences between VCs and CIPs and, instead, have attributed enhanced Fc effector responses in controllers to qualitative and not quantitative binding differences $(14,54)$. In these studies, qualitative features such as enhanced IgG3/IgG1 Ab subclass-mediated Fc effector responses and altered Ab Fc constant region glycosylation were associated with controller status.

In the RV144 HIV vaccine trial, non-neutralizing Abs that mediated ADCC correlated with protection from the acquisition of HIV-1 $(10,11)$. Interestingly, upon subsequent analysis, RV144 Abs possessing high ADCC activity were shown to target specific regions of $\operatorname{HIV} \operatorname{Env}(16,55,56)$. This may indicate that Abs, at least in part, can mediate enhanced levels of ADCC by targeting optimal regions of Env. In support of this concept, Bruel et al. recently demonstrated that bnAbs targeting different regions of HIV-1 Env, but possessing the same Fc constant region, yielded different levels of FCGR3A signaling and ADCC (57). Taken together, these data suggest that the region of Env that is targeted contributes to the efficiency of FCGR signaling, along with other qualitative $\mathrm{Ab}$ Fc constant region features like glycosylation and $\mathrm{Ab}$ subclass. Future studies are needed to characterize the qualitative factors that may modulate the efficiency of FCGR activation by VC IgG in our cohort.

We examined the ability of patient IgG to bind, neutralize, and mediate FCGR signaling against the same HIV-1 Env (NL4-3). We found no significant differences between the level of neutralization mediated by VC or CIP Abs. We also saw no differences in the levels of Ab binding when comparing VC or CIP Abs. In contrast, we observed that VC IgG possessed enhanced FCGR signaling in comparison with CIP. This suggests that the enhanced FCGR activation mediated by VC IgG is not associated with an enhanced capacity to bind or neutralize HIV-1. However, in examining CIP IgG, we found a significant correlation between FCGR3A signaling and neutralization (Figure 6D). Previous studies characterizing $\mathrm{Ab}$ responses against simian immunodeficiency virus (SIV) and HIV infections have noted that FCGR-mediated effector responses and neutralization can evolve separately. In SIV studies, 


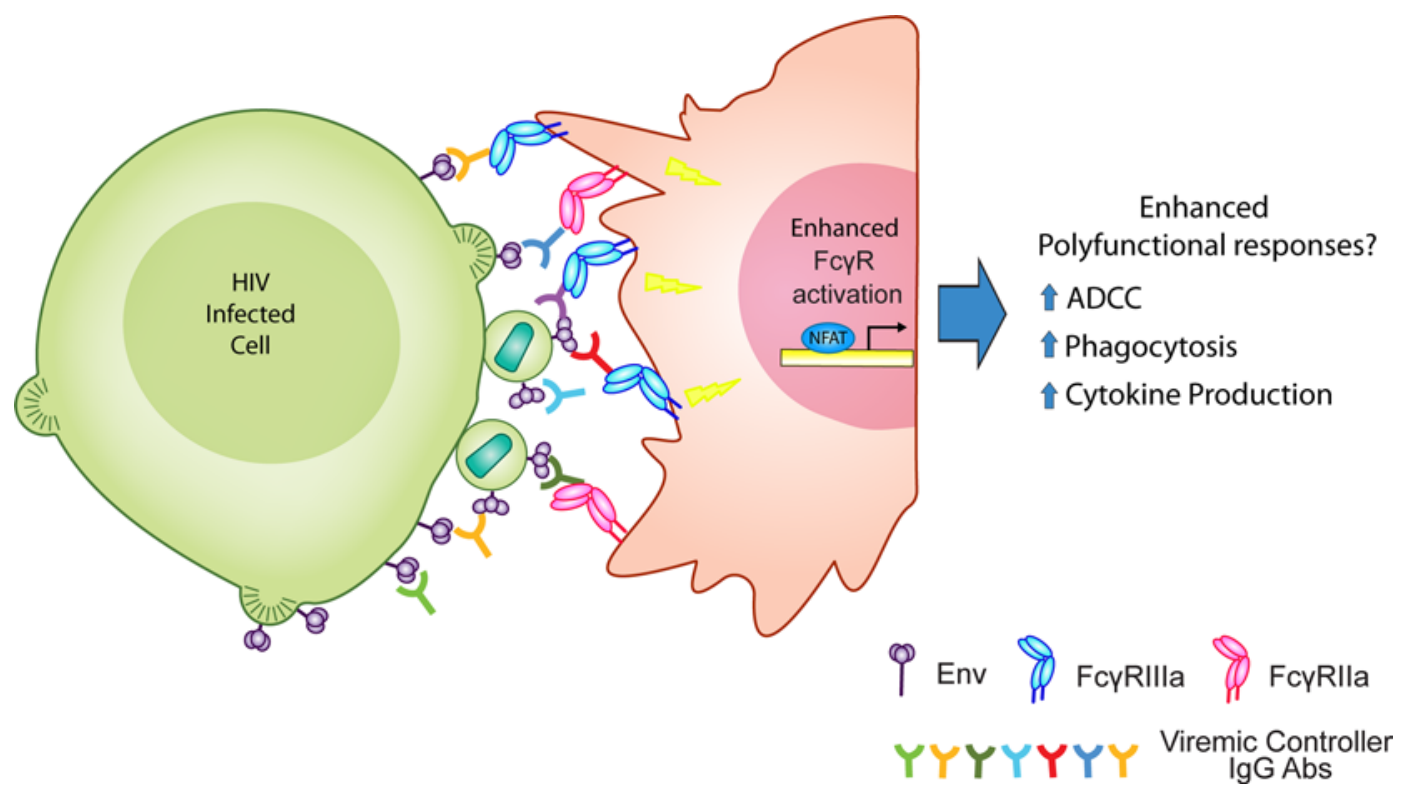

Figure 7. Working model for enhanced VC IgG FCGR activation. Diagram depicts the potential implication of enhanced viral controlers (VC) IgC activation.

IgG-induced Fc effector activities were observed during acute infection (weeks), while neutralizing responses were not detected until months after the initial infection (58). During acute HIV infection, a decline in IgG-induced FCGR effector responses coincided with the development of IgG neutralizing responses (59). Taken together with our data, these data suggest a complex relationship between the efficiency of FCGR signaling and neutralization, where in some cases the evolution of neutralizing responses may come at the expense of FCGR signaling activity.

We note that our study has exploited the robust Ab binding to the laboratory isolate NL4-3 Env, which is also highly sensitive to neutralization (Tier 1). Future work is needed to explore the relationship between the breadth of HIV-1 neutralization against a panel of more resistant primary Env isolates (Tiers 1-3), and the breadth and magnitude of FCGR activation.

In comparing the levels of FCGR2A and FCGR3A signaling for each IgG, we observed that the majority of VCs displayed a spectrum of enhanced FCGR activation profiles (Figure 6E). Recent studies have found that VC IgG possess a greater breadth of Fc effector responses as compared with CIP IgG (i.e., higher polyfunctionality) (14). Since the efficiency of Fc effector responses is dependent on the level of FCGR activation, we hypothesize that the enhanced FCGR activation stimulated by VC IgG may lead to the greater breadth of polyfunctional Fc-mediated responses observed in VCs (Figure 7) (14). Future studies are needed to characterize the breadth of VC IgG-mediated Fc effector responses induced in different innate effector cell types and to correlate this with particular FCGR signaling profiles.

In this study, we performed multivariate analysis to examine if the IgG functions we tested were predictive of patient groups. As a whole, these IgG parameters were significantly predictive of controller status $(P=0.036)$. However, when we conducted multivariate analysis using only the FCGR activation data, we observed that these parameters were more strongly predictive of controller status $(P=0.0018)$. Additionally, we performed an unbiased hierarchical clustering analysis and identified 2 clusters that were enriched with VCs, which were significantly distinct from other IgG profiles. These VC enriched profiles were characterized by high FCGR3A activation and low neutralization and Ab binding, or high uniform binding, neutralization, and FCGR activation (Figure 6G). A recent study examined the use of IgG Fc activity profiling as a methodology to define and characterize protective IgG responses in HIV vaccinees (60). This study examined 64 biophysical and functional IgG properties that included binding affinity, epitope diversity, and glycosylation. In our study, FCGR activation was highly predictive of patient group and, therefore, may aid in the rapid identification of IgG features associated with protective responses when coupled with more extensive biophysical and functional profiling. Future studies should aim to integrate FCGR signal profiling into more extensive profiling approaches. 
In light of several studies showing that patient HIV-specific Ab titers, neutralization, and ADCC are reduced in $\mathrm{HIV}^{+}$patients as measure of time on ART (40-44), we examined if ART in CIPs affected the mean distribution of any Ab parameters tested (Supplemental Figure 2). In this cohort, the time on ART did not appear to correlate with the mean distribution of any Ab parameters tested. In line with our findings, Ackerman et al. found no significant differences in the capacity of IgG from $\mathrm{HIV}^{+}$ patients on and off ART to mediate ADCC, phagocytosis, NK activation, or complement activation (14). Additionally, Jensen et al. observed no significant reduction in the capacity of IgG from HIV patient to mediate ADCC 6 months after the initiation of ART (61). Nonetheless, we do not have longitudinal measurements for the $\mathrm{Ab}$ activities measured for this study and, therefore, cannot rule out that the IgG activity for individual CIPs have not decreased as a measure of time on treatment. Nevertheless, if ART significantly affected our ability to compare Ab functions among VC and CIPs, we would likely observe global alterations in all of the Ab functions tested; however, we only observed significant differences in comparing VC versus CIP FCGR signaling (Figure 3 and 4) and not Ab binding or neutralization (Figure 2 and 5).

In addition to the effect of ART, we examined if CD4 cell counts correlated with IgG functions in CIPs. We observed no significant relationship between CD4 cell counts and IgG function (Supplemental Figure 3). However, studies have shown that CD4 cell nadirs were more highly associated with the modulation of $\mathrm{Ab}$ responses than current CD4 cell counts (46). Despite a rebound in CD4 T cell levels after HAART, a high proportion of HIV-infected individuals are unable to maintain protective Ab levels against vaccination antigens $(45,62,63)$. This defect was significantly correlated with low CD4 nadirs. It has been hypothesized that the permanent depletion or impairment of B and/or CD4 T cell subsets during chronic infection may cause the permanent dysregulation of $\mathrm{Ab}$ responses in patients with low CD4 nadirs (64). In our study, we observed that the IgG functions did not correlate with either time on HAART or current CD4 cell counts; however, since we do not have CD4 cell nadir count for the CIPs, we cannot discount that they have not affected the evolution of HIV-specific Ab responses. Since we did not observe differences in $\mathrm{CIP}$ versus $\mathrm{VC} \mathrm{Ab}$ binding or neutralization, it will be important to assess if low CD4 nadirs specifically affect the efficiency of IgG to mediate FCGR activation. This would be very important in the context of therapeutic vaccines, since defects in eliciting IgG-mediated FCGR activation due to low CD4 nadirs could hinder the efficacy of any vaccine strategy. Moreover, identifying the $\mathrm{T}$ and/or $\mathrm{B}$ cell subsets involved in maintaining IgG-mediated FCGR activation may yield critical insights into the evolution of these responses and aid in the development of an HIV vaccine.

Overall, this study finds that enhanced FCGR2A and/or FCGR3A signaling is a common feature associated with viremic control of HIV-1. Therefore, we believe that FCGR activation profiling may represent a rapid, simple, and reliable method for performing high-throughput IgG screens to assess the levels of $\mathrm{Ab}$ mediated HIV control in infected patients and Ab-mediated protection in HIV vaccinees.

\section{Methods}

IgG isolation from HIV-positive and $\mathrm{HIV}^{-}$patient sera. $\mathrm{HIV}^{+}$patients were recruited from the Jack Martin Fund Clinic at Mount Sinai Hospital, and plasma samples were obtained. Plasma samples were collected from 18 HIV VC, which consisted of 9 LTNPs whose viral load is < 1,000 copies/ml and 9 "elite" controllers whose viral loads are $<50$ copies/ml, as well as 35 HIV-1 CIPs on HAART and $5 \mathrm{HIV}^{-}$volunteers. Patient demographic characteristics are summarized in Table 1. Polyclonal IgG were isolated from plasma using a protein $\mathrm{A} / \mathrm{G}$ spin column kit, followed by desalting using Zeba spin columns according to manufacturer's instructions (Thermo Fisher Scientific). IgG yields were quantified using an Easy-Titer IgG assay kit (Thermo Fisher Scientific).

TZM-bl HIV-1 neutralization assays. Patient IgG Ab-mediated HIV-1 neutralization was examined using a standard TZM-bl neutralization assay (38). Donor-derived $\mathrm{HIV}^{+}$and $\mathrm{HIV}^{-} \mathrm{IgG} \mathrm{Abs}$ were examined using 10-point neutralization curves generated from 3-fold serial dilutions that started at a top concentration of $500 \mu \mathrm{g} / \mathrm{ml}$. Positive controls for this assay were the bNAb 2G12 (NIH AIDS Reagent Program; catalog 1476) or control pooled polyclonal HIV IgG antibodies (NIH AIDS Reagent Program; catalog 3957). The virus input was standardized to yield a value of 400,000 relative light units (RLUs). Ab dilutions and virus (X4-tropic; pNL4-3) were incubated for 1 hour at room temperature (RT) and then added to TZM-bl cells cultured in DMEM containing 10\% FCS and DEAE-dextran at a final concentration of $15 \mu \mathrm{g} / \mathrm{ml}$. The levels of HIV tat-dependent luciferase production was measured 48 hours after infection. 
Cell-based CANVI Env Ab binding assay. Tetherin ${ }^{\text {high }}$ CD4 Jurkat cells were infected with HIV-1 $\Delta$ vpu mCherry expressing reporter virus and purified by Ficoll Hypaque (GE Healthcare) gradient 24 hours after infection. The next day, cells were normalized to $20 \%-30 \%$ infected (cherry+) cells and incubated with patient IgG or control $\mathrm{Abs}$ at $4^{\circ} \mathrm{C}$. After primary $\mathrm{Ab}$ incubation, opsonized cells are washed and incubated with an APC-conjugated anti-human total IgG secondary $\mathrm{Ab}$ (Invitrogen, catalog A21445) at $4^{\circ} \mathrm{C}$. The levels of HIV-specific Ab binding were quantified by flow cytometry. BI values were calculated by setting a quadrant gate in which the $\mathrm{HIV}^{-}$(cher$\mathrm{ry}^{-}$) population accounts for less than $1.25 \%$ to exclude nonspecific background staining. The $\mathrm{HIV}^{-}$population serves as an internal negative control for the assay in case a given IgG possesses some level of non-HIV-specific $\mathrm{Ab}$ binding. From this gate, a percentage of $\mathrm{HIV}^{+}$cells that have HIV-specific Abs bound to their surface is calculated. Next, the MFI of IgG bound to the surface of $\mathrm{HIV}^{+}$cells is divided by the MFI of the IgG bound to the HIV- population. The MFI ratio is then multiplied by the percentage of cells with Ab bound to their surface to give a BI value. To ensure that the relative differences between patient-derived IgG were maintained, all IgG were tested in parallel on the same day for each replicate.

FCGR activation assay. FCGR2A and FCGR3A signaling was measured using a Jurkat cell-derived reporter cell line that contains an integrated NFAT-driven firefly luciferase reporter gene $(30,37)$. FCGR signaling activates the NFAT transcription factor, inducing expression of firefly luciferase driven by an NFAT responsive promoter. Tetherin ${ }^{\text {high }} \mathrm{CD} 4^{+}$lymphocytes infected with HIV-1 $\Delta$ vpu were purified by Ficoll Hypaque gradient, normalized to $20 \%-30 \%$ infected cells, and preincubated for 15 minutes with 10 -fold serial dilutions of patient-derived Abs that started at a top concentration of $250 \mu \mathrm{g} / \mathrm{ml}$. The Ab opsonized, HIV-infected target cells were then cocultured with the FCGR2A or FCGR3A reporter cell line at a 2:1 effector/target ratio for 16 hours. After 16 hours, the cells were lysed and the levels of firefly luciferase activity were determined using a luciferase assay kit (Promega). Reporter cells were cocultured with the infected target populations in the absence of $\mathrm{Ab}$ to provide background (Ab-independent) luciferase production, and these levels were subtracted from the signal to yield Ab-specific activation in RLUs.

Multivariable clustering analysis. Multiparametric analysis of all metrics was evaluated by hierarchical clustering using Gene E (http://www.broadinstitute.org/cancer/software/GENE-E). Clustering of patients and IgG properties were based on pair-wise Euclidean distance measurements. We also used the R Packaged "pvclust" to perform the hierarchical cluster analysis (39). For each cluster in hierarchical clustering, "pvclust" calculates $P$ values via multiscale bootstrap resampling. $P$ values of a cluster is a value between 0 and 1 , which indicates how strong the cluster is supported by the data. Pvclust provides 2 types of $P$ values: an AU (approximately unbiased) $P$ value and a BP (bootstrap probability) value. We used the 1,000 permutations to calculate the BP $P$ values.

Statistics. Statistical analysis of data was performed using Graph Pad PRISM Software. Significance between infected patient groups was calculated using a 2-tailed Mann-Whitney $U$ test. Correlations between the interrelatedness of 2 functions/parameters were calculated using Spearman rank tests. $P$ values of less than or equal to 0.05 were deemed significant. The mean \pm SEM is shown in graphs. We also performed univariate and multivariate regression analysis using linear models in $\mathrm{R}$. The $\mathrm{P}$ values for the multivariate analysis were calculated using MANOVA in R that performed the Hotelling-Lawley analysis to find the significance of the model.

Study approval. Mount Sinai's Human Research Protection Program IRB (New York City, New York, USA) approved the study ISMMS IBC protocol number HS\#: 11-01150. Informed consent was obtained from all research subjects prior to study participation.

\section{Author Contributions}

Project planning was done by RAA, AMM, BKC, VS, AFS. Experimental work was performed by RAA, AMM, NDD, MIB. Data analysis was performed by RAA, AMM and KL. Reagent development was done by RAA and AIW. Patient plasma acquisition was managed by VS, MTH, GRC, and DSF. The manuscript was written by RAA and BKC and the final version was approved by all authors. Data analyses were performed by RAA, AMM, MK, and KL. Reagent development was done by RAA, MT, and AIW.

\section{Acknowledgments}

This study was supported by NIH grant GM11388 and AI104471 to BKC; NIH/NIAID grants P01AI090935 and R01AI073450 to AFS; and NIH/NIAID grants R01AI064001, R01AI120998, and R56AI125173 to VS. RAA was awarded an NIH Loan Reimbursement grant to perform this work. We would like to thank Susan Zolla-Pazner and Caterina Hoie for their critical review of this manuscript. 
Address correspondence to: Raymond A. Alvarez or Benjamin K. Chen, 1425 Madison Avenue, Division of Infectious Diseases; Annenberg bldg 23-30; New York, New York 10029, USA. Phone: 212.241.2510. E-mail: Raymond.alvarez@mssm.edu (R.A. Alvarez); Benjamin.chen@mssm.edu (B.K. Chen).

1. Walker BD, Yu XG. Unravelling the mechanisms of durable control of HIV-1. Nat Rev Immunol. 2013;13(7):487-498.

2. Sáez-Cirión A, Pancino G. HIV controllers: a genetically determined or inducible phenotype? Immunol Rev. 2013;254(1):281-294

3. Baum LL, et al. HIV-1 gp120-specific antibody-dependent cell-mediated cytotoxicity correlates with rate of disease progression. J Immunol. 1996;157(5):2168-2173.

4. Lambotte $\mathrm{O}$, et al. Heterogeneous neutralizing antibody and antibody-dependent cell cytotoxicity responses in HIV-1 elite controllers. AIDS. 2009;23(8):897-906.

5. Wren LH, et al. Specific antibody-dependent cellular cytotoxicity responses associated with slow progression of HIV infection. Immunology. 2013;138(2):116-123.

6. Johansson SE, et al. NK cell function and antibodies mediating ADCC in HIV-1-infected viremic and controller patients. Viral Immunol. 2011;24(5):359-368

7. Ahmad R, et al. Evidence for a correlation between antibody-dependent cellular cytotoxicity-mediating anti-HIV-1 antibodies and prognostic predictors of HIV infection. J Clin Immunol. 2001;21(3):227-233.

8. Carrington M, Walker BD. Immunogenetics of spontaneous control of HIV. Annu Rev Med. 2012;63:131-145.

9. Poropatich K, Sullivan DJ. Human immunodeficiency virus type 1 long-term non-progressors: the viral, genetic and immunological basis for disease non-progression. J Gen Virol. 2011;92(Pt 2):247-268.

10. Haynes BF, et al. Immune-correlates analysis of an HIV-1 vaccine efficacy trial. N Engl J Med. 2012;366(14):1275-1286.

11. Rerks-Ngarm S, et al. Vaccination with ALVAC and AIDSVAX to prevent HIV-1 infection in Thailand. N Engl J Med. 2009;361(23):2209-2220.

12. Nimmerjahn F, Ravetch JV. Fcgamma receptors as regulators of immune responses. Nat Rev Immunol. 2008;8(1):34-47.

13. Nimmerjahn F, Ravetch JV. Antibody-mediated modulation of immune responses. Immunol Rev. 2010;236:265-275.

14. Ackerman ME, et al. Polyfunctional HIV-Specific Antibody Responses Are Associated with Spontaneous HIV Control. PLoS Pathog. 2016;12(1):e1005315

15. Chung AW, et al. Polyfunctional Fc-effector profiles mediated by IgG subclass selection distinguish RV144 and VAX003 vaccines. Sci Transl Med. 2014;6(228):228ra38.

16. Yates NL, et al. Vaccine-induced Env V1-V2 IgG3 correlates with lower HIV-1 infection risk and declines soon after vaccination. Sci Transl Med. 2014;6(228):228ra39.

17. Chung AW, et al. Immune escape from HIV-specific antibody-dependent cellular cytotoxicity (ADCC) pressure. Proc Natl Acad Sci USA. 2011;108(18):7505-7510.

18. Chung AW, Rollman E, Center RJ, Kent SJ, Stratov I. Rapid degranulation of NK cells following activation by HIV-specific antibodies. J Immunol. 2009;182(2):1202-1210.

19. Ferrari G, et al. An HIV-1 gp120 envelope human monoclonal antibody that recognizes a C1 conformational epitope mediates potent antibody-dependent cellular cytotoxicity (ADCC) activity and defines a common ADCC epitope in human HIV-1 serum. J Virol. 2011;85(14):7029-7036.

20. Fouda GG, et al. HIV-specific functional antibody responses in breast milk mirror those in plasma and are primarily mediated by IgG antibodies. $J$ Virol. 2011;85(18):9555-9567.

21. Gómez-Román VR, et al. A simplified method for the rapid fluorometric assessment of antibody-dependent cell-mediated cytotoxicity. J Immunol Methods. 2006;308(1-2):53-67.

22. Gómez-Román VR, et al. An adenovirus-based HIV subtype B prime/boost vaccine regimen elicits antibodies mediating broad antibody-dependent cellular cytotoxicity against non-subtype B HIV strains. J Acquir Immune Defic Syndr. 2006;43(3):270-277.

23. Isitman G, Chung AW, Navis M, Kent SJ, Stratov I. Pol as a target for antibody dependent cellular cytotoxicity responses in HIV-1 infection. Virology. 2011;412(1):110-116.

24. Kantakamalakul W, Pattanapanyasat K, Jongrakthaitae S, Assawadarachai V, Ampol S, Sutthent R. A novel EGFP-CEM-NK flow cytometric method for measuring antibody dependent cell mediated-cytotoxicity (ADCC) activity in HIV-1 infected individuals. J Immunol Methods. 2006;315(1-2):1-10.

25. Pollara J, et al. High-throughput quantitative analysis of HIV-1 and SIV-specific ADCC-mediating antibody responses. Cytometry A. 2011;79(8):603-612.

26. Jardetzky T. HIV: conformational camouflage. Nature. 2002;420(6916):623-624.

27. Kwong PD, et al. HIV-1 evades antibody-mediated neutralization through conformational masking of receptor-binding sites. Nature. 2002;420(6916):678-682.

28. Munro JB, et al. Conformational dynamics of single HIV-1 envelope trimers on the surface of native virions. Science. 2014;346(6210):759-763.

29. Smalls-Mantey A, et al. Antibody-dependent cellular cytotoxicity against primary HIV-infected CD4+ T cells is directly associated with the magnitude of surface IgG binding. $J$ Virol. 2012;86(16):8672-8680.

30. Alvarez RA, et al. HIV-1 Vpu antagonism of tetherin inhibits antibody-dependent cellular cytotoxic responses by natural killer cells. J Virol. 2014;88(11):6031-6046.

31. Arias JF, et al. Tetherin antagonism by Vpu protects HIV-infected cells from antibody-dependent cell-mediated cytotoxicity. Proc Natl Acad Sci USA. 2014;111(17):6425-6430.

32. Pham TN, Lukhele S, Hajjar F, Routy JP, Cohen ÉA. HIV Nef and Vpu protect HIV-infected CD4+ T cells from antibodymediated cell lysis through down-modulation of CD4 and BST2. Retrovirology. 2014;11:15.

33. Veillette M, et al. Interaction with cellular CD4 exposes HIV-1 envelope epitopes targeted by antibody-dependent cell-mediated 
cytotoxicity. J Virol. 2014;88(5):2633-2644.

34. Sánchez-Mejorada G, Rosales C. Signal transduction by immunoglobulin Fc receptors. J Leukoc Biol. 1998;63(5):521-533.

35. Pinter A, Honnen WJ, He Y, Gorny MK, Zolla-Pazner S, Kayman SC. The V1/V2 domain of gp120 is a global regulator of the sensitivity of primary human immunodeficiency virus type 1 isolates to neutralization by antibodies commonly induced upon infection. J Virol. 2004;78(10):5205-5215.

36. Walker LM, et al. Broad and potent neutralizing antibodies from an African donor reveal a new HIV-1 vaccine target. Science. 2009;326(5950):285-289.

37. Tada M, Ishii-Watabe A, Suzuki T, Kawasaki N. Development of a cell-based assay measuring the activation of Fc $\gamma$ RIIa for the characterization of therapeutic monoclonal antibodies. PLoS One. 2014;9(4):e95787.

38. Montefiori DC. Measuring HIV neutralization in a luciferase reporter gene assay. Methods Mol Biol. 2009;485:395-405

39. Suzuki R, Shimodaira H. Pvclust: an R package for assessing the uncertainty in hierarchical clustering. Bioinformatics. 2006;22(12):1540-1542.

40. Casazza JP, Betts MR, Picker LJ, Koup RA. Decay kinetics of human immunodeficiency virus-specific CD8+ T cells in peripheral blood after initiation of highly active antiretroviral therapy. J Virol. 2001;75(14):6508-6516.

41. Notermans DW, et al. Potent antiretroviral therapy initiates normalization of hypergammaglobulinemia and a decline in HIV type 1-specific antibody responses. AIDS Res Hum Retroviruses. 2001;17(11):1003-1008.

42. Morris L, et al. HIV-1 antigen-specific and -nonspecific B cell responses are sensitive to combination antiretroviral therapy. $J$ Exp Med. 1998;188(2):233-245.

43. Morris MK, Katzenstein DA, Israelski D, Zolopa A, Hendry RM, Hanson CV. Characterization of the HIV-1 specific humoral immune response during highly active antiretroviral therapy (HAART). J Acquir Immune Defic Syndr. 2001;28(5):405-415.

44. Madhavi V, et al. Antibody-dependent effector functions against HIV decline in subjects receiving antiretroviral therapy. $J$ Infect Dis. 2015;211(4):529-538.

45. Titanji K, et al. Loss of memory B cells impairs maintenance of long-term serologic memory during HIV-1 infection. Blood. 2006;108(5):1580-1587.

46. Cagigi A, Nilsson A, De Milito A, Chiodi F. B cell immunopathology during HIV-1 infection: lessons to learn for HIV-1 vaccine design. Vaccine. 2008;26(24):3016-3025.

47. Bournazos S, Klein F, Pietzsch J, Seaman MS, Nussenzweig MC, Ravetch JV. Broadly neutralizing anti-HIV-1 antibodies require Fc effector functions for in vivo activity. Cell. 2014;158(6):1243-1253.

48. Forthal DN, et al. Antibody-dependent cellular cytotoxicity independently predicts survival in severely immunocompromised human immunodeficiency virus-infected patients. J Infect Dis. 1999;180(4):1338-1341.

49. Forthal DN, Landucci G, Keenan B. Relationship between antibody-dependent cellular cytotoxicity, plasma HIV type 1 RNA, and CD4+ lymphocyte count. AIDS Res Hum Retroviruses. 2001;17(6):553-561.

50. Hessell AJ, et al. Fc receptor but not complement binding is important in antibody protection against HIV. Nature. 2007;449(7158):101-104.

51. Madhavi V, et al. Breadth of HIV-1 Env-specific antibody-dependent cellular cytotoxicity: relevance to global HIV vaccine design. AIDS. 2014;28(13):1859-1870.

52. Egan MA, Carruth LM, Rowell JF, Yu X, Siliciano RF. The ins and outs of HIV endocytosis. Trends Cell Biol. $1997 ; 7(2): 49$.

53. Checkley MA, Luttge BG, Freed EO. HIV-1 envelope glycoprotein biosynthesis, trafficking, and incorporation. $J$ Mol Biol. 2011;410(4):582-608.

54. Ackerman ME, et al. Natural variation in Fc glycosylation of HIV-specific antibodies impacts antiviral activity. J Clin Invest. 2013;123(5):2183-2192.

55. Bonsignori M, et al. Antibody-dependent cellular cytotoxicity-mediating antibodies from an HIV-1 vaccine efficacy trial target multiple epitopes and preferentially use the VH1 gene family. J Virol. 2012;86(21):11521-11532.

56. Zolla-Pazner S, et al. Vaccine-induced IgG antibodies to V1V2 regions of multiple HIV-1 subtypes correlate with decreased risk of HIV-1 infection. PLoS One. 2014;9(2):e87572.

57. Bruel T, et al. Elimination of HIV-1-infected cells by broadly neutralizing antibodies. Nat Commun. 2016;7:10844

58. Asmal M, et al. Antibody-dependent cell-mediated viral inhibition emerges after simian immunodeficiency virus SIVmac251 infection of rhesus monkeys coincident with gp140-binding antibodies and is effective against neutralization-resistant viruses. J Virol. 2011;85(11):5465-5475.

59. Dugast AS, et al. Independent evolution of Fc- and Fab-mediated HIV-1-specific antiviral antibody activity following acute infection. Eur J Immunol. 2014;44(10):2925-2937.

60. Chung AW, et al. Dissecting Polyclonal Vaccine-Induced Humoral Immunity against HIV Using Systems Serology. Cell. 2015;163(4):988-998.

61. Jensen SS, et al. HIV-specific ADCC improves after antiretroviral therapy and correlates with normalization of the NK cell phenotype. J Acquir Immune Defic Syndr. 2015;68(2):103-111.

62. Jordano Q, et al. Invasive pneumococcal disease in patients infected with HIV: still a threat in the era of highly active antiretroviral therapy. Clin Infect Dis. 2004;38(11):1623-1628.

63. Lange CG, et al. Nadir CD4+ T-cell count and numbers of CD28+ CD4+ T-cells predict functional responses to immunizations in chronic HIV-1 infection. AIDS. 2003;17(14):2015-2023.

64. French M, Keane N, McKinnon E, Phung S, Price P. Susceptibility to opportunistic infections in HIV-infected patients with increased CD4 T-cell counts on antiretroviral therapy may be predicted by markers of dysfunctional effector memory CD4 T cells and B cells. HIV Med. 2007;8(3):148-155. 